INRA Prod. Anim.,

2014, 27 (2), 77-88

\title{
L'innovation en élevage : de nouvelles démarches pour de nouveaux enjeux
}

\author{
J.-M. MEYNARD ${ }^{I}$, J.-Y. DOURMAD $D^{2,3}$ \\ ${ }^{1}$ INRA, département SAD, Campus de Grignon, F-78850 Thiverval-Grignon, France \\ 2 INRA, UMR1348 Pegase, F-35590 Saint-Gilles, France \\ 3 Agrocampus Ouest, UMR1348 Pegase, F-35000 Rennes, France \\ Courriel : jean-marc.meynard@grignon.inra.fr
}

Pour répondre aux enjeux du développement durable, l'élevage, comme l'agriculture en général, doit faire l'objet d'un important effort d'innovation. Quelle contribution peut-on attendre de la recherche à ce processus d'innovation?

Une intense activité d'innovation a marqué l'histoire de l'élevage ${ }^{1}$ : la domestication et la sélection des races ont permis depuis des millénaires de sécuriser et d'améliorer l'alimentation de l'homme et de lui fournir des forces de travail. Les pratiques d'élevage et d'alimentation des animaux ont constamment évolué, s'adaptant à la diversité des écosystèmes et des besoins des populations humaines, intégrant les progrès technologiques et agronomiques. Depuis les années 1950, on a assisté à une accélération de ce processus, parallèlement au développement de la recherche et à la mondialisation des connaissances. De nouveaux génotypes, produits vétérinaires, formulations d'aliments du bétail, ou équipements, ou encore de nouvelles pratiques et organisations d'élevages ont émergé et se sont diffusés. Alors qu'auparavant les innovations étaient avant tout conçues par des éleveurs pour des éleveurs, l'origine des innovations s'est diversifiée, avec une contribution importante des industries d'amont et d'aval. Ces innovations visent aujourd'hui à répondre non seulement aux attentes des éleveurs et des filières, mais aussi à celles de la société. Des attentes nouvelles émergent de la part d'une plus large diversité d'acteurs nécessitant des changements parfois radicaux de pratiques d'élevage ou de systèmes de production (Meynard et al 2012). Dans un monde qui change très rapidement, les éleveurs doivent impérativement innover, que ce soit pour anticiper ou pour s'adapter, pour rester compétitifs ou pour survivre. Parallèlement, la question de l'acceptabilité des innovations se pose de plus en plus fréquemment, aussi bien du fait des éleveurs que de la société. Les citoyens se sentent de plus en plus concernés par l'agriculture, ses produits et ses manières de produire. La globalisation des échanges et des développements technologiques amplifie les impacts de certaines innovations, qui peuvent changer radicalement les conditions de production, comme les conditions de vie des acteurs du monde agricole et des territoires. Ainsi, le développement de variétés de soja tolérantes aux herbicides et l'accroissement de la demande mondiale en protéines, ont entraîné, en Amérique du Sud, l'extension du soja au détriment des prairies et de l'élevage bovin extensif, et ont accéléré la financiarisation de l'agriculture induisant une évolution profonde des unités de production et une déstructuration des sociétés rurales (Albaladejo 2012). Si une innovation est économiquement rentable à court terme et bénéficie de puissants moyens de diffusion, elle peut être adoptée à très grande vitesse ; si elle a des impacts sociaux ou environnementaux négatifs, ceux-ci peuvent rapidement devenir préoccupants et nourrir sa contestation. Les organismes génétiquement modifiés, les pesticides ou certaines formes d'élevage industriel sont ainsi devenus les symboles d'une agriculture " productiviste " rejetée par une partie de nos concitoyens.

Dans ce contexte complexe, où l'innovation est considérée par les uns comme un enjeu économique majeur et scrutée par les autres comme potentiellement porteuse de risques inacceptables, les scientifiques et les ingénieurs sont directement interpellés. Comment juger de la pertinence d'une innovation ? Comment aider à l'émergence d'innovations pertinentes? Comment concevoir une innovation, et quelles connaissances mobiliser pour favoriser la conception ?
Comment anticiper les bénéfices qu'on peut en attendre et les risques qu'elle pourrait induire ? Comment favoriser la diffusion des innovations les plus intéressantes ? Ces questions ont reçu des réponses variées dans le domaine de l'élevage, mais aussi dans d'autres secteurs économiques. L'objectif de cet article, en introduction à un numéro spécial de la revue INRA Productions Animales dédié à l'innovation dans les systèmes d'élevage, est de tenter un rapide tour d'horizon de ces questions, en nous appuyant sur ces différents acquis.

\section{1 / L'innovation en élevage}

\section{1 / La diversité du processus d'innovation et de ses résultats}

Si l'on s'en réfère la définition qui en est donnée par l'Union Européenne (COM 2003) « une innovation consiste à produire, assimiler et exploiter avec succès une nouveauté dans le monde économique ou social ». Une autre définition, plus économique, proposée par la Chambre des Lords (House of Lords 2010) dans son rapport sur l'innovation en agriculture se réfère à « un processus qui permet de produire une valeur ajoutée par l'application de connaissances dans un domaine où elles ne l'étaient pas jusqu'à présent ». Une invention originale ou des idées nouvelles ne sont donc pas suffisantes. Pour qu'il y ait innovation, il faut qu'elles rencontrent une demande et que des utilisateurs se les approprient.

Si l'on regarde l'innovation en élevage au travers de l'image qui en est donnée

${ }^{1}$ Comme le soulignent Fagerberg et al (2005) « Où en serions-nous sans l'innovation fondamentale qu'a constituée l'agriculture ?» 
dans les revues agricoles ou les salons professionnels, on constate tout d'abord l'importance qu'elle occupe dans la communication des entreprises du secteur de l'élevage. Les principaux salons professionnels nationaux et internationaux consacrés à l'élevage construisent ainsi une part importante de leur communication autour de l'innovation, par exemple en attribuant des labels ou des prix « innovation ». Ces innovations concernent principalement des installations pour l'hébergement des animaux, des équipements pour la mise en œuvre des différentes opérations d'élevage et pour la gestion des effluents, de nouveaux aliments ou suppléments nutritionnels et dans une moindre mesure des outils d'aide à la décision et à la gestion des élevages. Ces innovations, avant tout technologiques, visent généralement à améliorer la productivité de l'élevage, à faciliter la vie de l'éleveur, ou à s'adapter à l'évolution des réglementations et à la demande sociétale, en particulier dans les domaines de l'environnement, du bien-être animal et de la qualité des produits. Elles s'inscrivent dans une vision positive du progrès technique, intégrant aujourd'hui certains aspects du développement durable. Paradoxalement, l'amélioration génétique des animaux est rarement présentée en tant qu'innovation, mais plutôt comme un processus d'amélioration sur le long terme. Ce sont plutôt les nouvelles méthodes de sélection, comme la sélection génomique, où les technologies de reproduction développées pour la sélection, comme le transfert d'embryons, qui constituent les innovations. De même

\section{Tableau 1. Les innovations en élevage. Différentes dimensions pour les caractériser}

\begin{tabular}{l}
\hline Domaine d'application : Bâtiments ; Équipements d'élevage ; Animaux ; Santé \\
animale ; Reproduction ; Gestion d'élevage ; Alimentation animale ; Production et \\
conservation des fourrages ; Gestion des effluents ; Organisation de l'élevage ; \\
Organisation de l'exploitation ; Organisation de la filière ; Organisation du \\
paysage \\
\hline Nature de l'objet innovant : Équipements ou matériels ; Intrants (produits \\
vétérinaires; aliments, semences...) ; Pratiques d'élevage ; Race animale \\
améliorée ; Produits animaux transformés ou non ; Outils de gestion ou d'aide à la \\
décision ; Système fourrager ; Système d'élevage ; Système d'exploitation ; \\
Mosaïque paysagère ; Filière de diversification \\
\hline Finalité : Productivité du troupeau ; Revenu ; Qualité ou quantité de travail ; \\
Emploi ; Environnement ; Santé et Bien-être animal ; Qualité des produits ; Image \\
de la production ; Autonomie énergétique, protéique et/ou décisionnelle de \\
l'exploitation \\
\hline Origine de l'innovation : Éleveurs ; Groupements d'éleveurs ; Entreprises \\
d'amont ; Entreprises d'aval ; Bureaux d'étude ; Organismes de Recherche et de \\
Développement (INRA, Instituts techniques, Chambres d'agriculture, CIVAM...); \\
et, très souvent, partenariats entre différents acteurs \\
\hline Originalité de l'innovation par rapport à l'existant : Innovation incrémentale ; \\
Innovation radicale ou de rupture
\end{tabular}

une entreprise d'amont et un organisme de recherche, l'innovation est plutôt de nature incrémentale. La construction d'une filière d'élevage " label rouge » constitue plus une innovation de rupture avec comme finalités l'accroissement du revenu des producteurs, l'amélioration de la qualité du produit et de l'image de la production; les objets innovants sont le produit commercialisé (des poulets « label », par exemple), le système d'élevage et la nouvelle filière, le domaine d'application concerne pratiquement l'ensemble du système de production et l'organisation de la filière ; les groupes d'éleveurs sont principalement à l'origine de l'innovation avec le contribution de la recherche-développement pour sa formalisation (Marousseau 2012).

\section{2 / Les forces motrices de l'in- novation en élevage}

Meynard et al (2006, 2012) identifient quatre moteurs principaux du changement et de l'innovation en agriculture et en élevage.

\section{a) L'évolution de la demande alimentaire}

Guillou et Matheron (2011) mettent en avant la nécessité, pour satisfaire les besoins d'une population croissante, d'accroître la disponibilité de produits alimentaires au niveau mondial. L'extension des terres agricoles étant limitée, il est envisagé pour cela de mobiliser différents leviers : accroissement de la productivité ${ }^{2}$, réduction des pertes et des gaspillages, accroissement de la contribution nutritionnelle des protéines végétales au détriment des protéines animales... toutes ces modifications sont susceptibles d'affecter fortement les modes de production. Le Ministère français de l'Agriculture ${ }^{3}$ affiche ses priorités : «Nourrir une population mondiale en expansion nécessite un accroissement de la production alimentaire fondée sur une augmentation de la productivité de l'agriculture. Cet objectif impose cependant de concilier durablement les performances économiques, sociales et environnementales ". Dans ce contexte, une désintensification, pour raison environnementale, à un endroit du monde peut conduire ailleurs à une intensification ou à de la déforestation, pour satisfaire la demande alimentaire mondiale. Cet objectif quantitatif s'accompagne d'une forte évolution concernant la qualité des produits. Les marchés, autrefois régis autour de conventions sectorielles portant sur une qualité standard, sont de plus en plus segmentés

\footnotetext{
2 Toutefois pour l'Europe, où de nombreux systèmes agricoles sont déjà fortement intensifiés et productifs, il n'est pas certain que l'accroissement de la productivité soit souhaitable partout, et il convient de s'interroger sur « le coût (économique, énergétique et environnemental) des derniers quintaux » (Meynard et al 2009), comme du porcelet ou du litre de lait supplémentaire.

3 http://agriculture.gouv.fr/L-agroecologie-une-voie-d-avenir
} 
(Allaire 2002). Les entreprises de collecte, les industries de transformation et les distributeurs multiplient les contrats imposant au produit des critères de qualité spécifiques, ou aux pratiques de culture et d'élevage des cahiers des charges contraignants. Les enjeux de la qualification ne concernent plus seulement les caractéristiques substantielles du produit, mais aussi la définition et la maîtrise de ses conditions d'obtention (origine, manières de produire ; Casabianca et Matassino 2006). La vive sensibilité des consommateurs à la qualité sanitaire des produits conduit à s'interroger sur les modalités de maîtrise des maladies des végétaux et animaux. Le développement du végétarisme et l'émergence de mouvements de défense des animaux, remettant en cause les conditions d'élevage industriel, sont aussi des moteurs d'innovation et d'évolution des pratiques.

b) La maîtrise des nuisances environnementales et la protection des ressources non renouvelables

Selon le « Millenium Ecosystem Assessment » (2005), l'agriculture porte une responsabilité particulière dans la dégradation de la qualité des milieux aquatiques (pollutions par nitrates, phosphore, pesticides), des sols (érosion, contaminations par des pesticides ou des métaux lourds), et de l'air (pesticides, gaz à effet de serre), mais aussi dans l'érosion de la biodiversité et dans la consommation de ressources non renouvelables (pétrole, phosphates, potassium). Le rapport de la FAO «Livestock long shadow » (Steinfeld et al 2006) réalise un diagnostic convergeant, pointant particulièrement l'émission de méthane par les ruminants et la déforestation, contribuant à l'effet de serre. Ces impacts s'expriment au niveau global, comme au niveau local. Ainsi, par exemple, les systèmes d'élevage intensifs européens peuvent contribuer localement à des pollutions de l'eau et de l'air, et favorisent en Amérique du Sud le développement de la culture du soja, dont les impacts écologiques (déforestation, homogénéisation des mosaïques paysagères, pollutions par les pesticides) sont maintenant bien connus (Botta et al 2011, Salembier et Meynard 2013). La réduction de ces nuisances et pollutions est d'ores et déjà intégrée dans les processus d'innovation actuellement à l'œuvre, mais souvent de manière secondaire et limitée. Pour que la dimension environnementale devienne un moteur majeur de l'innovation, des évolutions politiques et institutionnelles seront indispensables, ainsi que le souligne le « Millenium Ecosystem Assessment ».

\section{c) Travail des agriculteurs et formes d'agriculture}

La mondialisation des échanges, qui induit en Europe une forte fluctuation des prix des produits agricoles, se traduit par une recomposition des choix productifs et de la combinaison des activités dans les exploitations. Les systèmes spécialisés se développent aux dépens des systèmes de polyculture-élevage ${ }^{4}$ (Schott et al 2010). L'accroissement de la taille des exploitations et de la surface par travailleur, ainsi que le développement de la pluriactivité des ménages et des agriculteurs eux-mêmes, appellent à une réorganisation des modes de production qui est souvent source d'innovations. Le travail constitue un point clé de cette réorganisation, à la fois source de différentiels de compétitivité avec les concurrents internationaux et contrainte ou moteur majeur dans l'évolution des systèmes techniques (Madelrieux et Dedieu 2008). Pour assurer la compétitivité et la pérennité des exploitations, il faudra innover en matière d'organisation du travail, promouvoir des systèmes agricoles flexibles et réactifs (Astigarraga et Ingrand 2010, Dedieu et al 2011), capables de s'adapter à une instabilité des cours des produits agricoles ou de l'énergie, et à un accroissement des risques climatiques (Martin 2006). Enfin, le développement de l'agriculture financière renouvèle en profondeur les débats sur les modèles d'agriculture de demain, concernant particulièrement le rapport au temps (vision d'entreprise et recherche de profits ou de rentabilité de court terme $v s$ gestion « en bon père de famille » sur le plus long terme) et au travail (des coûts de production à réduire $v s$ une ressource à valoriser au mieux).

\section{d) Evolution de la place de l'agriculture dans les territoires}

Dans un contexte de marginalisation du rôle économique et social de l'agriculture au niveau de nombreux territoires en Europe, les agriculteurs sont perçus comme étant investis d'une mission de gestion de l'espace et des ressources naturelles, et plus seulement de production. On parle à cet égard de multifonctionnalité de l'agriculture. Selon les caractéristiques des territoires, se posent des questions spécifiques : quelle agriculture, quel élevage en zone périurbaine, en fonction des opportunités et des contraintes liées à la proximité de la ville et à la forte densité de population non agricole (Soulard et Thareau 2009) ? Quels systèmes de culture ou d'élevage dans les zones écologiques protégées, où la « production de naturel » peut devenir prioritaire par rapport à la production de denrées (Bellon et al 2012) ? Quelles combinaisons d'activités dans les exploitations agricoles de montagne ou les régions à fort potentiel touristique, entre la production de denrées, la vente directe, l'accueil à la ferme, le travail extérieur ? Les activités agricoles doivent organiser leur coexistence et leur complémentarité avec les autres usages de l'espace, avec les autres activités rurales et avec la qualité du cadre de vie des résidents et touristes. La transformation des systèmes agricoles intéresse donc directement les acteurs non agricoles du territoire, avec lesquels des compromis, des arrangements ou des synergies doivent être recherchés (Etienne 2010). La construction des mosaïques paysagères, la gestion des services écosystémiques, la valorisation des ressources patrimoniales (races locales ou savoir-faire par exemple), ou la mise en place de nouvelles relations entre producteurs et consommateurs renvoient à un travail de conception d'agroécosystèmes (Berthet 2013).

Derrière ces différentes forces motrices, se dessinent potentiellement des évolutions radicales des systèmes agricoles: ainsi, l'internalisation des attentes de la société vis-à-vis de l'environnement pourrait entraîner en Europe une remise en cause des fondamentaux de l'agriculture actuelle tels que l'usage massif des produits vétérinaires et des pesticides ${ }^{5}$, l'intégration croissante des activités par l'aval des filières, ou la spécialisation des exploitations et des territoires. On pourrait aussi assister à une différenciation beaucoup plus poussée qu'aujourd'hui entre, par exemple, $i$ ) une agriculture des territoires périurbains, marquée par les contraintes de la cohabitation avec les résidents mais aussi par le développement de circuits courts, ii) une agriculture de zones à forte valeur patrimoniale ou récréative, où la priorité pourrait être donnée à la production de paysages et à la protection de la biodiversité, et iii) une agriculture visant avant tout compétitivité et productivité, tournée vers l'agro-industrie et/ou l'exportation : les différenciations territoriales des systèmes agricoles s'accompagneraient alors d'une différenciation de leurs critères d'évaluation.

Cependant, d'une manière générale, au-delà de tendances globales comme l'accroissement de l'importance accordée à la gestion des ressources environnementales, il est difficile d'anticiper l'évolution, à dix ou quinze ans, du contexte économique international, des politiques

\footnotetext{
${ }^{4}$ On notera, cependant, qu'à l'échelle de certaines régions une diversité relative des productions animales et végétales persiste, malgré une spécialisation croissante des exploitations.

${ }^{5}$ Les démarches « écophyto » et « écoantibio » initiées en France à la suite du Grenelle de l'environnement s'inscrivent déjà dans cette perspective.
} 
publiques, des mouvements d'opinion et des rapports de force entre groupes de pression. De même, si l'on admet généralement la réalité du changement climatique en cours, ses répercussions locales restent incertaines. Faire un choix de priorités entre enjeux, pour concevoir ou évaluer des innovations, c'est privilégier un avenir, soit que l'on estime plus probable, soit que l'on juge souhaitable au regard d'un projet politique ou de l'insuffisance des solutions existantes.

\section{3 / Le positionnement du cher- cheur}

Dès lors que l'on admet, comme nous y invite, par exemple, la Stratégie Nationale de Recherche et d'Innovation ${ }^{6}$, que le rôle des chercheurs dans l'innovation n'est pas seulement d'apporter des connaissances (briques élémentaires) aux acteurs économiques innovants, mais également d'être acteurs du processus d'innovation, il convient de s'interroger sur les formes de cette participation et sur la nature des relations entre les chercheurs et les utilisateurs potentiels de l'innovation. Meynard et al (2006) identifient quatre postures différentes, caractérisant la contribution du chercheur à l'innovation dans le domaine agronomique :

- Il peut être à l'origine de l'invention, qui, une fois diffusée, appropriée, utilisée deviendra une innovation.

- Il peut proposer aux acteurs des outils et méthodes pour innover par euxmêmes (par exemple en matière de systèmes d'élevage), ou pour adapter à leur propre situation des innovations exogènes.

- Il peut contribuer à identifier, analyser, améliorer et promouvoir des innovations conçues par des acteurs de terrain (les agriculteurs et les éleveurs sont très inventifs).

- Il peut aider à anticiper les effets économiques, sociaux et environnementaux des innovations, les conditions de leur diffusion et les conséquences de cellesci sur les performances de l'agriculture et de l'élevage, à différentes échelles. L'enjeu est alors de savoir apprécier le plus précocement possible non seulement ce qu'on peut espérer gagner, mais aussi ce que l'on risque de perdre.

Ces différentes postures donnent une place plus ou moins grande à l'analyse et l'évaluation des systèmes agricoles existants, depuis la situation où c'est l'analyse de la réalité qui permet de hiérarchiser les éléments du système à améliorer, jusqu'à la situation, où par volonté de rupture, le chercheur choisit de s'abstraire de l'existant (par exemple, la conception de systèmes d'élevage innovants à partir de résultats de simulations). Dans la plupart des cas, le chercheur collabore, de manière plus ou moins étroite, avec des acteurs directement intéressés par les résultats de ses recherches. C'est ce que soulignait en 2005 la lettre de mission adressée par la Direction de l'INRA aux rédacteurs du rapport « recherches sur la conception de systèmes agricoles innovants "), qui indiquait « (la recherche) doit évidemment se réaliser en étroite coopération avec ces acteurs, seul gage de réussite de l'appropriation des résultats » (cité par Meynard et al 2006).

Analysant une base de données des inventions et innovations issues de l'INRA, et visant à améliorer les performances environnementales des exploitations agricoles, Coulon et Meynard (2011) notent que dans les filières animales, plus de $60 \%$ de celles-ci ont été conçues dans le cadre d'un partenariat avec des organismes de développement (instituts techniques avant tout), avec l'industrie (sélection et alimentation principalement) ou, de manière moins fréquente, avec des associations environnementales. Si plus de la moitié visent à augmenter l'efficience de certains intrants ou à leur substituer des ressources moins rares, près d'un tiers s'attache à proposer de nouveaux systèmes de culture ou d'élevage intégrant des modifications parfois profondes de leur fonctionnement $^{7}$. Cette proportion est bien supérieure à celle observée dans les publications techniques : par exemple, pour la réduction de l'usage des pesticides, l'essentiel des solutions proposées dans la presse agricole nationale concerne l'amélioration de l'efficience des intrants, la reconception de systèmes atteignant à peine 15\% (Butault et al 2010). « Cette différence, indiquent Coulon et Meynard (2011) est vraisemblablement liée au fait qu'un organisme de recherche comme l'INRA dispose de plus de liberté d'exploration de solutions très innovantes et de changements radicaux, que les instituts techniques ou les organismes de développement, qui, du fait de leurs missions, sont tenus de travailler sur des solutions immédiatement opérationnelles. En proposant ainsi une palette très large de solutions innovantes, et particulièrement des options de recon- ception de systèmes, l'INRA contribue à ouvrir le champ des possibles, et prépare d'autres avenirs que la poursuite des évolutions tendancielles».

La diversité des acteurs, des avenirs possibles et des situations locales multiplient à l'infini le besoin d'innovation. Les chercheurs doivent reconnaître que les priorités ne sont pas les mêmes pour tous, qu'elles ne sont pas les mêmes partout, et travailler à aider chacun à trouver sa propre solution, plutôt que de se mettre en quête d'innovations consensuelles et passe-partout, ou de systèmes agricoles idéaux. Dans la suite de cet article, nous tenterons de brosser les avancées récentes et les orientations actuelles concernant la recherche sur l'innovation en élevage, dans deux domaines majeurs : $i$ ) la conception des innovations (participation des chercheurs à la conception, outils et méthodes pour favoriser la conception par les acteurs : postures 1 et 2); ii) l'analyse in situ des innovations, de leurs performances et des conditions de leur diffusion (postures 3 et 4 ).

\section{2 / Concevoir des innova- tions : application aux sys- tèmes d'élevage}

L'innovation en élevage, comme dans les autres secteurs, est le plus souvent un processus collectif et interactif; il ne faut pas voir celui-ci comme un processus linéaire, où se succèderaient des étapes de recherche, de conception, de développement, de production et de diffusion, mais comme résultant d'allersretours entre ces activités. Pour autant, Le Masson et al (2006) montrent que les capacités d'innovation d'une structure ou d'un collectif sont fortement conditionnées par la gestion de ses activités de conception (le « moment » où émerge et se précise le concept de l'innovation), et l'organisation de ses relations avec la recherche et le développement. Cette partie s'attache à préciser les modalités que peut prendre la conception de systèmes d'élevage innovants, en s'appuyant à la fois sur les acquis des sciences zootechniques et sur les travaux théoriques en sciences de la conception.

\section{1 / Développer des processus de conception innovante}

Le Masson et al (2006) distinguent deux régimes de conception : la conception réglée («rule-based design ») et

\footnotetext{
6 SNRI http://www.enseignementsup-recherche.gouv.fr/pid24538/strategie-nationale-de-recherche-et-d-innovation-s.n.r.i.html

7 En référence à la typologie dite ESR - pour Efficience, Subtitution, Reconception - des voies utilisables pour la réduction des intrants de Hill et Mac Rae (1995), discutée par Lamine et Bellon (2009).
} 
la conception innovante (« innovative design »).

Le mode de conception le plus courant est la conception réglée. L'objectif de la conception est alors d'améliorer, de manière graduelle, des produits ou technologies existants. Les objectifs de la conception ne changent pas et peuvent donc être clairement définis à l'avance. Les compétences mobilisées et les modalités d'évaluation des résultats de la conception (prototypes, essais, tests, indicateurs) n'ont donc pas besoin d'être changés. Cette stabilité permet de développer des méthodes de travail standardisées, qui permettent de réduire les coûts de conception et de développement de l'innovation. Dans le domaine de l'élevage, la conception réglée peut être illustrée par les progrès incrémentaux réalisés depuis 40 ans dans la manière de calculer des rations alimentaires ou de composer des aliments du bétail. Dans l'objectif, poursuivi de manière continue, d'affiner l'équilibre des apports et d'améliorer leur biodisponibilité, cette démarche s'est appuyée sur les progrès dans la connaissance des matières premières, la mise au point de nouvelles manières d'évaluer la valeur des aliments (INRA-AFZ 2004), ou la mise au point de nouveaux ingrédients comme les acides aminés ou les enzymes. Des modèles informatisés et des outils d'aide à la décision en alimentation animale (INRAtion ${ }^{\circledR}$, InraPorc ${ }^{\circledR}$, Partur'in ${ }^{\circledR}$ ) ont été construits, pour automatiser et simplifier le calcul. Les chercheurs ont fortement contribué à ce processus de conception, en apportant aux différentes étapes de nouvelles connaissances, qui ont été intégrées dans le calcul des rations sans en changer le principe, ou en développant des outils d'aide à la décision.

Cependant, la conception réglée n'est plus adaptée lorsque les cadres de la conception sont remis en cause. La conception innovante devient alors nécessaire. Elle désigne un processus d'exploration de nouveaux « possibles » visant à satisfaire des attentes tout à fait différentes. Souvent, ces attentes ne sont pas complètement spécifiées au début de la conception, et se précisent au fur et à mesure que l'objet conçu prend forme. Il n'est alors pas possible de spécifier à l'avance les compétences requises pour conduire le processus de conception, ni les méthodes d'évaluation. La conception innovante demande non seulement de la créativité, mais aussi une grande capacité à faire évoluer au cours du processus, au fur et à mesure que le concept de l'innovation se précise, les objectifs visés, ainsi que les champs de savoir, les connaissances et les collaborations mobilisées. La question de l'association des utilisateurs à la conception est centrale : comme les objectifs précis de la conception, ainsi que les modalités d'évaluation ne sont pas donnés d'avance, il est important de mobiliser très tôt les utilisateurs pour qu'ils contribuent à faire émerger ceux-ci en cours de conception. La conception innovante pose ainsi de manière particulièrement aigüe la question de l'intégration d'une anticipation des usages dans la conception (Cerf et al 2012).

Dans le domaine agricole, les forces motrices de l'innovation que nous avons rappelées plus haut justifient un effort sans précédent de conception innovante.

- De nouveaux objectifs prennent de l'importance, en particulier la montée en puissance de préoccupations concernant l'environnement et le bien-être animal (Dourmad et al 2006). Les approches au niveau de l'animal ou de la parcelle, qui avaient jusqu'ici dominé la recherche ne sont plus suffisantes, l'innovation devant prendre en compte des processus qui ne prennent sens qu'au niveau du troupeau (bien-être animal, économie, travail) ou du paysage (services écosystémiques liés à la biodiversité, Dumont et al 2013). Comme le soulignent Bos et al (2012), l'accroissement du nombre de parties prenantes, d'acteurs ayant de bonnes raisons de participer à la reconception des systèmes agricoles, y compris ceux extérieurs au monde de l'élevage, rend particulièrement complexe la fixation de ces objectifs.

- La nature des connaissances mobilisées pour la conception évolue elle aussi. En ouvrant le champ des possibles, la conception innovante implique des questions nouvelles et la mobilisation de nouveaux champs de connaissances. En recherche, les spécialistes de l'animal (physiologistes, zootechniciens), classiquement en première ligne pour la conception d'innovations en élevage, doivent s'adjoindre les compétences d'éthologues, d'écologues (Dumont et al 2013), de spécialistes de l'analyse des risques (pour gérer l'obligation de résultats, mise en avant dans les contrats portant sur la qualité ou l'environnement), de spécialistes du travail et de l'évolution des métiers (ergonomes ou sociologues). Le changement climatique remet aussi en question l'expérience et les références des acteurs de terrain, qui, pour concevoir des innovations pertinentes pour le futur doivent intégrer les savoirs issus de modèles climatologiques.

- L'évaluation des concepts d'innovations est aujourd'hui enrichie de nombreux critères sociaux (durée et pénibilité du travail, acceptabilité) et, surtout, environnementaux, qui se rajoutent aux critères économiques classiques. Des méthodes spécifiquement adaptées aux nouveaux enjeux, et à leur complexité sont développées : analyse de cycle de vie, analyse multicritère, etc. (cf. le $\mathrm{n}^{\circ}$ spécial de la revue en ligne Innovations agronomiques consacré à l'évaluation agri-environnementale, 2013). L'évaluation des capacités d'adaptation des systèmes d'élevage innovants dans un environnement incertain devient essentielle : en quoi les systèmes préserventils des marges de manœuvre, réserventils des possibilités d'ajustement des itinéraires de production (Dedieu et Servière 2011) ? Des méthodes d'évaluation participative, associant l'ensemble des parties prenantes et mobilisant d'autres critères que ceux que les chercheurs jugent importants, deviennent indispensables.

On peut assez facilement trouver des innovations pouvant se rattacher à un processus de conception innovante. Par exemple, dans le domaine de l'élevage porcin, la mise au point dans les années 1970 de la conduite en bande des troupeaux de truies a "révolutionné » la conduite des élevages en considérant simultanément les dimensions de gestion de la reproduction, d'organisation de la production, de planification du travail, de conception des bâtiments et de gestion de la santé. Elle a pour origine la rencontre entre un ingénieur de l'Institut Technique du porc (aujourd'hui IFIP), qui avait eu l'expérience de la conduite de très grands troupeaux dans les pays de l'Est avec un éleveur des Côtesd'Armor qui se posait la question de l'organisation de son élevage de quelques dizaines de truies. La recherche-développement a ensuite formalisé la technique en mobilisant de nombreuses disciplines, zootechnie, reproduction, santé animale, ingénierie des bâtiments et équipements d'élevage, économie, etc. (Le Denmat et al 1984). Le développement dans le cadre du Réseau Agriculture Durable (RAD) de systèmes laitiers plus autonomes valorisant l'herbe constitue un autre exemple qui illustre bien la démarche de ce type d'innovation (Alard et al 2002).

Les exemples d'innovations issues d'un processus de conception innovante impliquant d'emblée une large diversité d'acteurs (éleveurs, recherche développement, société, consommateurs, distributeurs, administration...) sont moins nombreux et plus récents. On peut citer le cas du développement d'un nouveau système de production d'œufs au PaysBas. Le système a été co-construit sur une " page blanche " à l'aide d'une approche holistique considérant simultanément différentes dimensions de l'élevage (bien-être animal, environnement, performance, image...) dans une perspective de long terme (Groot Koerkamp et Bos 2008), en allant jusque la construction 
d'un prototype commercial (www. rondeel.org). La conception pas-à-pas de systèmes laitiers autonomes combinant des approches expérimentales en station et un réseau d'exploitation de terrain constitue un autre exemple de conception innovante, avec une démarche différente (Coquil et al 2009).

Dans le paragraphe qui suit, nous nous intéresserons particulièrement à la conception innovante de systèmes d'élevage, domaine en profonde mutation, sous l'effet d'avancées méthodologiques récentes et du développement des approches d'agro-écologie et/ou d'écologie industrielle (Dumont et al 2013, Thomas et al 2014 ce numéro).

\section{2 / La conception innovante de systèmes d'élevage : conception de novo et conception pas à pas}

La conception de systèmes d'élevage ne peut pas être enfermée dans une démarche normative par laquelle il faudrait obligatoirement passer (Dedieu et Servière 2011). Entreprendre un travail de conception suppose d'adapter sa démarche au cas à traiter et au contexte de la conception, en empruntant aux travaux antérieurs avec pragmatisme et rigueur. Meynard (2008, 2012) relève que les démarches de conception de systèmes agricoles innovants s'organisent en deux grandes familles d'approches : l'approche "de novo » et l'approche « pas à pas » (tableau 2) :

La conception « de novo » vise à déterminer des systèmes performants (en référence aux nouveaux objectifs) sans se préoccuper, au moins dans un premier temps, de la transition, de la manière de passer du système actuel à l'innovant. On privilégie l'invention, la rupture. Les ateliers de conception (Bos et al 2009, Reau et al 2012, Agogué et al 2013) offrent des conditions très favorables à la conception de novo, et permettent de bien valoriser la variété des savoirs, associés à des métiers ou à des disciplines variés, et leur complémentarité. L'utilisation de modèles agronomiques ou zootechniques constitue également un moyen très efficace pour faire de la conception de novo : ils permettent une exploration très large des combinaisons de techniques, bien au-delà de ce que les meilleurs experts connaissent, et informent le concepteur sur le comportement à long terme des systèmes qu'il imagine. Citons par exemple le modèle « Mélodie » dans le domaine des impacts environnementaux des élevages (Chardon et al 2012) ou le modèle "Sows " dans le domaine de la conduite des élevages porcins (Martel et al 2008). De tels modèles permettent d'identifier, parmi une multitude de possibilités, les combinaisons de choix techniques qui remplissent un cahier des charges donné, en termes de production, revenu, travail ou impacts environnementaux. Les travaux actuels mettent l'accent sur la manière de mobiliser les savoirs non scientifiques dans la conception de novo. Martin et al (2012) ont ainsi conçu un « rami fourrager » pour la conception de scénarios de systèmes fourragers avec des éleveurs et des conseillers. Gouttenoire et al (2013) proposent une démarche de modélisation participative, associant les éleveurs candidats à la modification de leur système d'élevage ; des modèles qualitatifs, prenant la forme de « cartes de relations causales » permettent de mettre en évidence l'importance, pour les performances du système d'élevage, de processus organisationnels peu étudiés par les chercheurs. Bos et al (2009) ont développé une méthodologie appelée RIO (acronyme néerlandais pour Conception Réflexive et Interactive) qui associe des éleveurs, des acteurs de la filière, des associations environnementalistes, des consommateurs, pour co-concevoir simultanément bâtiments d'élevage, systèmes d'élevage et produits innovants.

Dans la conception pas à pas, on cherche avant tout à organiser le changement. Un système existant sert de point de départ ; il est graduellement modifié en s'appuyant sur des boucles d'apprentissage (Meynard 2008, Coquil et al 2009), pour aboutir à un système innovant qui n'était pas connu à l'avance. Le travail de conception débute par un diagnostic (le système de production peut-il être jugé satisfaisant ?). Le diagnostic permet d'identifier les points clés du système d'élevage à améliorer (quels indicateurs ne sont pas à un niveau satisfaisant ? quels processus sont en jeu ?), et d'imaginer les modifications de pratiques ou d'organisation susceptibles d'améliorer la situation. Après mise en œuvre de ces changements, un nouveau diagnostic est réalisé, qui permet de vérifier si la situation a été améliorée, et de déterminer de nouvelles priorités : c'est une véritable boucle d'amélioration continue qui se met en route. Par rapport à la conception de novo, l'exploration est plus prudente, mais présente l'avantage de s'adapter aisément aux contraintes spécifiques de chaque situation agricole. La conception pas à pas se prête bien à une mobilisation progressive des agriculteurs ou des éleveurs, dans une démarche de développement. Le producteur, souvent accompagné par un technicien ou par un collectif de pairs, met au point, année après année, son nouveau système, en même temps qu'il apprend à le piloter, se convainc de son intérêt, et réorganise progressivement son travail et ses moyens de production (Mischler et al 2009).

Pour la mise à l'épreuve des systèmes conçus de novo, comme pour celle de la démarche de conception pas à pas, une étape de test expérimental est souvent jugée utile. Outre l'amélioration des systèmes et des démarches, ce test permet de produire des références sur les performances des systèmes, sur les risques qu'ils induisent, et contribue à susciter la confiance des acteurs dans les résultats de la conception, sans prise de risque jugée inacceptable par des agriculteurs. On parle alors d'expérimentation-système, puisque ce sont des systèmes de production qui y sont testés. Alors que dans l'expérimentation

Tableau 2. Les deux familles d'approches pour la conception innovante de systèmes de culture ou d'élevage.

\begin{tabular}{|l|l|l|}
\hline & Conception de novo & Conception pas à pas \\
\hline Principe général & $\begin{array}{l}\text { Invention d'un système en rupture par } \\
\text { rapport à l'existant }\end{array}$ & $\begin{array}{l}\text { Evolution progressive d'un système } \\
\text { existant }\end{array}$ \\
\hline Conduite de la transition & Non & Oui \\
\hline Méthodes & $\begin{array}{l}\text { Explorations à l'aide de modèles } \\
\text { agronomiques ou zootechniques ; } \\
\text { Ateliers de conception participative }\end{array}$ & $\begin{array}{l}\text { Boucle d'amélioration continue : } \\
\text { diagnostic du système actuel, } \\
\text { identification de solutions, mise en } \\
\text { œuvre, nouveau diagnostic }\end{array}$ \\
\hline Avantages & $\begin{array}{l}\text { Exploration de solutions très innovantes ; } \\
\text { source d'inspiration pour la conception } \\
\text { pas à pas. }\end{array}$ & $\begin{array}{l}\text { Apprentissage progressif des } \\
\text { nouveaux systèmes; adaptation aux } \\
\text { contraintes spécifiques de la ferme }\end{array}$ \\
\hline Risques & Faible réalisme & Conservatisme \\
\hline
\end{tabular}


factorielle, on compare les effets de choix techniques élémentaires (par exemple génotype, régime alimentaire, traitement vétérinaire), l'expérimentation-système vise à comparer les performances des systèmes innovants aux objectifs en fonction desquels ils ont été conçus (Meynard et al 1996). Ces expérimentations peuvent être mises en œuvre dans une ferme expérimentale, dans des réseaux de parcelles agricoles ou au niveau de fermes entières. Les expérimentations-systèmes réalisés en production végétale ont le plus souvent été conduites à l'échelle de la parcelle (Debaeke et al 2009), parfois à l'échelle de l'exploitation. Les expérimentations réalisées en élevage s'adressent à l'échelle de l'atelier ou du troupeau, ou à celle de l'exploitation agricole. Elles relèvent le plus souvent de démarches de conception de novo, et visent à vérifier comment un système inventif conçu à partir de la combinaison de connaissances scientifiques et empiriques fonctionne à une échelle pluriannuelle ou en situations d'aléas climatiques et économiques, voir par exemple Dedieu et al (2008) et Benoit et al (2009) pour des systèmes ovins extensifs et biologiques, Germain et al (2013) pour les systèmes de poulet de chair biologique, Lebret et al (2011) et Dourmad et al (2009) pour les systèmes alternatifs d'engraissement de porcs. Mais on assiste depuis plusieurs années à l'émergence d'expérimentations systèmes basées sur le principe de la conception pas à pas, par exemple lorsqu'il s'agit de développer de façon progressive et réflexive un système allant vers une autonomie croissante (en polyculture élevage, voir Coquil et al 2009).

Conception pas à pas et conception de novo font en partie appel aux mêmes ressources méthodologiques de base : les modèles agronomiques, dont le rôle est majeur dans la conception de novo, peuvent aussi être mobilisés pour le diagnostic dans la conception pas à pas. Les indicateurs agro-écologiques sont utilisés pour l'évaluation de systèmes candidats (conception de novo), aussi bien que pour nourrir le diagnostic dans des démarches pas à pas. Enfin, les deux approches peuvent être combinées : dans un travail de conception pas à pas au niveau de l'exploitation, Mischler et al (2009) montrent que des itinéraires techniques du blé conçus de novo peuvent être incorporés comme innovation « élémentaire " au cours d'une boucle d'amélioration. Coquil et al (2009) mettent en expérimentation des systèmes de polyculture élevage conçus de novo, et les améliorent progressivement dans une démarche pas à pas. On voit aujourd'hui se diversifier les démarches de conception innovante de systèmes agricoles, preuve de la vitalité de ce domaine scientifique.

\section{3 / La conception innovante au niveau du territoire}

Une action au niveau des exploitations agricoles individuelles est évidemment insuffisante pour gérer des processus fortement dépendants de la structure des paysages (évolution de la biodiversité, pollutions d'aquifères...) et de réseaux d'acteurs inscrits dans les territoires locaux. Elle doit s'accompagner d'une conception de mosaïques paysagères (Vasseur et al 2012), de modalités d'agencement spatial des systèmes agricoles (Thenail et al 2009) ou d'agro-écosystèmes (Berthet 2013), impliquant différents acteurs du territoire.

Comment cette conception collective peut-elle être organisée ? Il s'agit d'une question complexe, qui n'a été abordée que récemment par la recherche. En effet, les intérêts des différents acteurs peuvent être contradictoires, leurs représentations de la situation inconciliables, ou leur information asymétrique ; certains peuvent ne retirer aucun bénéfice d'une coordination des actes gestionnaires, ou ne pas percevoir les bénéfices qu'ils pourraient en retirer (Meynard et Casabianca 2011). Pour de telles questions les démarches participatives sont particulièrement adaptées, car elles sont favorables aux processus d'apprentissage qui accompagnent la conception.

Berthet (2013), qui analyse la conception de mosaïques paysagères favorables à la biodiversité, et plus particulièrement à la protection d'espèces d'oiseaux des plaines, fait des propositions originales concernant la gestion collective d'un fonds écologique. Pour désamorcer d'éventuels conflits entre des acteurs privilégiant des fonctions productives et d'autres privilégiant des fonctions environnementales de l'agriculture, elle propose de considérer le fonds écologique comme un « inconnu commun »: les acteurs seront d'autant plus ouverts à des solutions originales que le bien commun à gérer ne sera pas défini au départ, mais le résultat d'une construction collective. Ainsi, que le montre Berthet (2013), la conception d'innovations territoriales résulte de la conception de plusieurs innovations, à différentes échelles : par exemple, les écologues ayant montré l'importance, pour les espèces d'oiseaux à protéger, des surfaces en prairies et de leur répartition dans le paysage (Bretagnolle et al 2011), trois objets ont été conçus pour mettre cette idée en application : $i$ ) des itinéraires techniques de la luzerne permettant de concilier production de fourrage et protection de la biodiversité, ii) une filière de foin de luzerne, gérée par une coopérative, destinée à approvisionner des éleveurs, iii) des contrats de production de luzerne, permettant d'étaler sa distribution dans le paysage afin de maximiser sa contribution à la préservation de la biodiversité. De même, des approches de modélisation (Sabatier et al 2012) ou des approches expérimentales (Farrugia et al 2012) ont permis d'explorer des stratégies d'utilisations innovantes des prairies en termes de densité animale et de période de fauche ou de pâturage, favorisant la biodiversité (oiseaux, insectes, flore) tout en préservant un niveau de production élevé.

La conception collective est également au cœur des travaux du groupe ComMod ${ }^{8}$ (Etienne et al 2005). Il s'agit, le plus souvent, de concevoir des modalités innovantes de gestion collective de ressources territoriales, ce qui nécessite une concertation entre des acteurs ayant au départ des visions très différentes de cette question. De manière très simplifiée, la démarche peut être résumée en trois étapes (Etienne 2010) : i) construire une représentation partagée des processus territoriaux qu'il s'agit de piloter : l'ensemble des parties prenantes (y compris les chercheurs) développent un modèle informatique qui rend compte des interactions clés entre les systèmes écologiques, agricoles et sociaux ; ii) engager un jeu de rôles, qui, en mettant les acteurs en situation, leur permet d'appréhender les conséquences de leurs choix individuels ou des scénarios de gestion collective qu'ils imaginent; iii) faire évoluer, d'un tour de jeu de rôle au suivant, les stratégies adoptées par certains acteurs ou les réglementations, comparer les scenarios ainsi construits, et progressivement, construire des règles de gestion collective donnant satisfaction à tous. Le modèle co-construit permet ainsi de peser les avantages et inconvénients des différents scenarios, de les améliorer, de construire des apprentissages collectifs et de se rapprocher de solutions satisfaisantes pour tous. Cette démarche a par exemple été utilisée pour rechercher des méthodes de gestion de la forêt associant propriétaires forestiers et éleveurs (Simon et Etienne 2010) ou pour étudier la co-évolution des territoires et des systèmes d'élevage extensif dans différents pays, en France, au Sénégal, au Brésil, en Uruguay et en Argentine (Leclerc et al 2010).

D’une manière générale, dans de telles démarches de conception, les apprentissages collectifs (nouvelles manières de

8 « Companion Modeling » pour modélisation d'accompagnement. 
penser, nouvelles formes de collaboration) réalisés au cours de la conception sont aussi importants que les résultats de la conception elle-même (Bos et al 2009) : ils forgent une culture commune à l'ensemble des acteurs concernés qui prépare ceux-ci aux étapes suivantes : gérer ensemble les objets conçus et, si nécessaire, les faire évoluer.

\section{3 / L'analyse in situ des inno- vations, de leurs performan- ces et des conditions de leur diffusion}

Ce dernier chapitre adopte un tout autre point de vue sur l'innovation : celleci est supposée conçue, testée, appropriée par au moins quelques pionniers. Il s'agit alors d'en analyser in situ les performances (bénéfices qu'en retirent les adoptants, effets non intentionnels) et d'éclairer les conditions de son adoption par de nouveaux acteurs.

\section{1 / Bénéfices attendus et rapidité de diffusion}

La rapidité de la diffusion des innovations dépend en partie de leur nature et de leur origine. La diffusion des innovations issues de conception réglée est généralement plus simple dans la mesure où il s'agit d'améliorer, de manière graduelle, des produits ou technologies existantes. Les nouvelles technologies viennent remplacer ou compléter les anciennes sans fondamentalement remettre en cause le système de production. En élevage bovin et ovin, Ingrand et al (2012) soulignent l'importance de l'amélioration du revenu ( $70 \%$ des réponses) et de la réduction de la durée et de la pénibilité du travail (65\% des réponses) dans les raisons citées par les éleveurs pour mettre en place une innovation. La diffusion de ce type d'innovation peut même parfois être « transparente » pour l'éleveur. C'est le cas par exemple des innovations en terme de formulation des aliments, qu'il s'agisse de nouveaux concepts ou de nouveaux ingrédients.

La diffusion d'innovations conçues pour répondre à des évolutions réglementaires relatives au bien-être animal ou à l'environnement est généralement largement déterminée par le rythme de mise en œuvre de ces réglementations. Le premier bénéfice attendu est alors de pouvoir continuer à produire dans le cadre du nouveau contexte réglementaire. De nombreux exemples récents existent dans le domaine du bien-être des animaux, avec l'interdiction des cages pour les poules pondeuses, l'interdiction de l'élevage en case individuelle des truies gestantes et des veaux de bou- cherie. Ceci peut conduire à des options techniques parfois très différentes selon les éleveurs, certains cherchant à privilégier les systèmes les plus proches possibles de ce qu'ils connaissent, par exemple la conduite des veaux ou des truies en petits groupes, alors que d'autres changent de paradigme, par exemple l'élevage en très grand groupe associé à des systèmes d'alimentation ou d'allaitement automatisés. Les raisons de cette diversité des choix ont été peu étudiées. Commandeur et al (2006) soulignent l'importance du « style d'élevage » et de l'espace d'information de l'éleveur. Ainsi, l'aversion au changement ou l'incompréhension de la rationalité de ces changements de la part des éleveurs et/ou de leurs conseillers constitue sûrement un frein à des évolutions plus radicales.

\section{2 / Diversité des exploitations agricoles, typologies d'adoptants}

Toute innovation ne concerne pas tous les agriculteurs. Dès la fin des années 1970 , partant du postulat que « les agriculteurs ont de bonnes raisons de faire ce qu'ils font $"$, plusieurs équipes du département SAD (à l'époque « Systèmes Agraires et Développement ») de l'INRA ont développé des démarches dites « d'approche globale des exploitations agricoles », visant à analyser la cohérence des choix productifs des agriculteurs, et ses implications pour l'adoption des innovations exogènes. Sebillotte (1974), Petit (1975) ou Osty (1978) montrent que l'analyse des logiques d'action des agriculteurs permet de comprendre pourquoi certains agriculteurs ne sont pas intéressés par certaines innovations, que les chercheurs considéraient pourtant faites pour eux : par exemple, des innovations exigeantes en temps de travail à un moment précis de l'année ne sont pas jugées pertinentes par les agriculteurs dont le système de production induit une pointe de travail à ce moment-là. Mais constater a posteriori ne suffit pas : pour prévoir quels agriculteurs sont susceptibles d'être intéressés par telle innovation, différentes approches prédictives ont été développées. Les typologies d'exploitations (Capillon et al 1988, Perrot et Landais 1993) ou d'éleveurs (Commandeur et al 2006), qui agrègent des fermes dont les stratégies productives se ressemblent, ont été proposées pour aider à adapter au cas par cas les conseils délivrés par des organismes de développement agricole. Plus récemment, Joannon (2004) a proposé un modèle d'analyse des marges de manœuvre des agriculteurs vis-à-vis de l'adoption de certaines innovations, et l'a appliqué au cas de la prévention de l'érosion.

Aujourd'hui, ces approches peuvent toujours être mobilisées dans des démarches de développement ou d'ex- pertise, mais elles ne constituent plus des fronts de recherche. La diversité des exploitations agricoles n'est plus vue comme une source d'obstacles à des innovations exogènes, mais comme la source d'une diversité d'innovations endogènes. Les agriculteurs ont toujours " de bonnes raisons de faire ce qu'ils font " et face à des difficultés spécifiques ou à une évolution de leur projet de vie, ils innovent. Les systèmes herbagers autonomes, la monotraite, la conduite en bandes des truies ou l'élevage de poulets label rouge constituent des exemples emblématiques d'innovations imaginées par des agriculteurs, et seulement ensuite étudiées par des chercheurs. Comme le soulignent Salembier et Meynard (2013), on considère de plus en plus souvent ces innovations de terrain comme des ressources pour favoriser une évolution plus massive des pratiques agricoles. Repérer les innovations de terrain, les évaluer pour déterminer les plus intéressantes (impacts sur les critères ciblés par l'agriculteur-concepteur, mais aussi sur les critères non cibles), et définir leurs conditions d'extrapolation, constitue une manière de construire des références sur les systèmes innovants. Des exemples d'une telle " traque aux systèmes innovants $\gg$ en production animale sont donnés par Ingrand et al (2012) ou par Salembier et Meynard (2013). Ces derniers montrent sur le cas de la Pampa Argentine que des systèmes innovants basés sur l'association polycultureélevage permettent de réduire fortement l'usage des pesticides et les nuisances environnementales des productions de grande culture. Ces travaux récents montrent l'importance, pour une évaluation in situ des innovations, de prendre en compte non seulement des critères standards (batteries d'indicateurs prédéfinis), mais également des critères liés aux enjeux personnels et aux valeurs des acteurs de terrain, essentiels pour comprendre la cohérence des systèmes pratiqués.

\section{3 / Verrouillage technologique et transitions sociotechniques}

Le concept de verrouillage technologique vise à expliquer la difficulté, voire l'impossibilité, du développement de technologies innovantes pourtant performantes : la technologie en place est devenue un tel standard pour la société qu'il semble difficile d'en changer. Plus précisément, le verrouillage conduit à un tri entre les innovations : celles qui sont totalement compatibles avec la technologie standard ont une chance de se développer, alors que celles qui remettent en cause soit celle-ci, soit les relations entre acteurs telles qu'elles se sont organisées autour du standard, ont beaucoup moins de chances de se développer (processus de dépendance au chemin, " path dependency »). 
Les recherches sur le verrouillage se sont d'abord développées dans d'autres secteurs que l'agriculture (le cas des claviers de machine à écrire est bien connu, David 1985). En agriculture, plusieurs cas d'étude, dans différents pays, ont démontré la puissance explicative de ce cadre d'analyse. Ainsi, un verrouillage autour de l'usage des pesticides a été mis en évidence en Europe et aux USA, qui retarde, voire bloque, le développement de solutions alternatives (Lamine et al 2010). Il est clair, bien que ce cas ne semble pas avoir été étudié de manière approfondie, qu'un verrouillage similaire existe en élevage dans le domaine de l'utilisation des antibiotiques ou plus généralement de la médication. Un verrouillage a également été identifié autour de la spécialisation des territoires vers l'agriculture ou l'élevage, entraînant la disparition de la polyculture-élevage (Schott et al 2010) : la concentration géographique des industries de transformation (qui simplifie leur logistique d'approvisionnement), la spécialisation géographique de l'élaboration de références et du conseil technique, la construction de connaissances empiriques et d'institutions dédiées et la spécialisation des chercheurs eux-mêmes (la céréaliculture et l'élevage spécialisés sont l'objet de beaucoup plus de travaux que la polyculture-élevage) contribuent à ce verrouillage. Le verrouillage technologique ne résulte pas d'une stratégie délibérée de tel ou tel acteur, mais des mécanismes d'auto-renforcement qui se créent autour d'une solution technologique (les pesticides, les produits vétérinaires, la spécialisation des exploitations et des territoires dans les exemples ci-dessus). Les principaux mécanismes d'auto-renforcement sont, d'après Farès et al (2012), les rendements croissants d'adoption, la compatibilité technologique, l'état de la connaissance et la structure organisationnelle des acteurs. Les économies d'agglomération contribuent également au renforcement de la concentration géographique des systèmes d'élevages, même en présence de contraintes environnementales, comme cela a été montré dans le cas de la production porcine en France (Gaigné et al 2012). Plus le nombre d'utilisateurs d'une technologie est élevé, plus elle configure la structure des réseaux d'acteurs et les apprentissages qui s'y déroulent. Plus une technologie est répandue, plus des technologies complémentaires se développent, renforçant sa position dominante. Les parcours de formation des acteurs (agriculteurs, conseillers, acteurs des filières et de la recherche-développement dans notre cas) influencent fortement leur capacité à utiliser telle ou telle technologie : chacun choisit celle qui lui semble la meilleure, compte tenu de ce qu'il sait. Ces effets cumulatifs augmentent ainsi la valeur d'adoption de la technologie initialement choisie.

Les travaux actuels s'orientent vers la mise au point de stratégies de " déverrouillage », en s'appuyant sur la théorie multiniveau des transitions sociotechniques de Geels (2002). Par exemple, Meynard et al (2013) mettent en évidence un verrouillage autour de la domination des tourteaux de soja dans la formulation des aliments composés pour le bétail (ruminants et volailles principalement). Une augmentation de l'autonomie de la France en protéines pour l'alimentation animale suppose de conduire des actions coordonnées adressées à différents acteurs, visant à la fois à soutenir la production de protéagineux, mais aussi à accroître la coordination entre les acteurs de la filière et à favoriser l'innovation variétale, agronomique et technologique.

\section{Conclusion}

Marquées depuis toujours par une intense activité d'innovation, les pratiques d'élevage ont constamment évolué pour s'adapter aux besoins croissants des populations humaines. Depuis les années 1950, on a assisté à une accélération de ce processus, parallèlement au développement de la recherche et à la mondialisation des connaissances. Cette évolution rapide a favorisé une homogénéisation des systèmes et des pratiques d'élevage qui a pu dans certains cas conduire à des situations de verrouillage et à des « impasses technologiques».

L'origine des innovations en élevage est très diverse avec une contribution importante de la recherche, du développement et des industries d'amont et d'aval, et une utilisation intensive de connaissances et d'informations. Mais il s'agit le plus souvent d'innovations visant à améliorer, de manière graduelle, des produits ou technologies déjà existantes, alors que dans le même temps des attentes nouvelles émergent de la part d'une plus large diversité d'acteurs nécessitant des changements plus radicaux de pratiques d'élevage ou de systèmes de production. Un effort important de conception innovante apparaît indispensable.
Pour les chercheurs, revisiter ainsi les chemins de l'innovation suppose un changement assez radical de posture (Hubert et al 2012). Il ne s'agit plus seulement de concevoir des innovations les mieux adaptées à une demande clairement exprimée par un partenaire, qui se chargera ensuite de leur diffusion : il s'agit de problématiser le besoin d'innovation, en s'interrogeant sur les normes et les critères d'efficacité généralement admis, pour les remettre en débat ; il s'agit d'éclairer les enjeux des différents choix, en contribuant à ouvrir une diversité de voies ; il s'agit de s'impliquer dans des processus collectifs, en acceptant le jeu d'une « reconstruction » simultanée des savoirs des chercheurs et de ceux des acteurs. A la lumière de ce triptyque " problématiser / éclairer / interagir » (Hubert et al 2012), le travail de conception apparaît indissociable d'un travail sur les savoirs (production de connaissances scientifiques, traque aux systèmes innovants chez des éleveurs), autant que d'une analyse du système sociotechnique dans lequel l'innovation est susceptible de trouver sa place.

Toutefois, la temporalité de la conception innovante apparaît mal adaptée à la dynamique et au mode de fonctionnement de projets de courte durée, de 3 à 5 ans, tels que ceux le plus couramment financés dans les programmes publics de soutien à la recherche et à l'innovation. Du fait des spécificités de la conception innovante, il peut être difficile de fixer à l'avance des "délivrables " ou de définir a priori le collectif des équipes impliquées. Les travaux sur l'organisation de la conception innovante dans les entreprises montrent que si la conception doit être fortement liée à la fois à la recherche et au développement, elle ne doit être inféodée ni à l'un ni à l'autre (Le Masson et al 2006). Deux obstacles symétriques peuvent brider la conception innovante : i) les réticences de responsables du développement agricole à voir leurs agents s'investir dans des innovations qui ne sont pas immédiatement rentables, dans les conditions techniques et économiques actuelles, ce qui restreint l'exploration de solutions potentiellement intéressantes ; ii) l'idée, encore partagée par de nombreux chercheurs, que l'innovation découle naturellement du progrès des connaissances scientifiques, alors que l'expérience de la conception innovante, tant en agriculture que dans l'industrie, montre que l'activité collective de conception doit piloter, au moins partiellement, l'acquisition des connaissances sur les processus. 


\section{Références}

Agogué M., Arnoux F., Brown I., Hooge S., 2013 Introduction à la conception innovante. Eléments théoriques et pratiques de la théorie C-K. Presses des Mines, Paris, France, 60p.

Alard V., Béranger C., Journet M., 2002. A la recherche d'une agriculture durable. Etude de systèmes herbagers économes en Bretagne, INRA Editions, Paris, France, 346p.

Albaladéjo C., 2012. Les transformations de l'espace rural pampéen face à la mondialisation. Annales de géographie, 686, 387-409.

Allaire G., 2002. L'économie de la qualité, en ses secteurs, ses territoires et ses mythes. Géogr., Econ., Soc., 4, 155-180.

Astigarraga L., Ingrand S., 2010. Production flexibility in extensive beef farming systems. Ecology and Society, 16, 7. [online: http:// www.ecologyandsociety.org/vol16/iss1/art7/]

Bellon S., Hemptinne J.L., 2012. Redefining frontiers between farming systems and the environment. In: Darnhofer I., Gibbon D. Dedieu B. (Eds). Farming Systems Research into the $21^{\text {st }}$ century: The new dynamic. Dordrecht: Springer, 307-333

Benoit M., Tournadre H., Dulphy J.P., Laignel G., Prache S., Cabaret J., 2009. Is intensification of ewe reproduction rhythm sustainable in an organically managed sheep production system? A 4-year interdisciplinary study. Animal, 3, 753-763.

Berthet E., 2013. Contribution à une théorie de la conception des agro-écosystèmres : Fonds écologique et inconnu commun. Thèse Ecole Nationale Supérieure des Mines de Paris, France, 232p.

Bos A.P., Groot Koerkamp P.W.G., Gosselink J.M.J., Bokma S.J., 2009. Reflexive interactive design and its application in a project on sustainable dairy husbandry systems. Outlook Agricult., 38, 137-145.

Bos A.P., Spoelstra S.F., Groot Koerkamp P.W.G., De Greef K.H., Van Eijk O.N.M., 2012. Reflexive design for sustainable animal husbandry: mediating between niche and regime. In: Spaargaren G., Loeber A., Oosterveer P. (Eds). Food practices in transtion. Changing food consumption, retail and production in the age of reflexive modernity. London: Routledge, 229-256.

Botta G.F., Tolón-Becerra A., Lastra-Bravo X., Tourn M.C., 2011. A Research of the Environmental and Social Effects of the Adoption of Biotechnological Practices for Soybean Cultivation in Argentina. Am. J. Plant Sci., 2, 359-369.

Bretagnolle V., Gauffre B., Meiss H., Badenhausser I., 2011. The role of grassland areas within arable cropping systems for the conservation of biodiversity at the regional level. Grassland Prod. Ecosys. Serv. Lemaire G., Hodgson J.A., Chabbi A. (Eds). CABI, 251-260.

Butault J.P., Dedryver C.A., Gary C., Guichard L., Jacquet F., Meynard J.M., Nicot P., Pitrat M., Reau R., Sauphanor B., Savini I., Volay T., 2010. Ecophyto R\&D, Quelles voies pour réduire l'usage des pesticides. Synthèse du rapport d'étude. INRA éditeur, France, 90 pages.

Capillon A., Legendre J., Simier J.P., Vedel G., 1988. Typologies et suivis technico-économiques d'exploitations: quels apports pour l'améliora- tion des systèmes fourragers? Fourrages, 115, 273-295.

Casabianca F., Matassino D., 2006. Local resources and typical animal products. In Rubino R., Sepe L., Dimitriadou A., Gibon A. (Eds). Livestock farming systems: Product quality based on local resources leading to improved sustainability. EAAP publication Nr. 118, 9-26.

Cerf M., Jeuffroy M.H., Prost L., Meynard J.M., 2012. Participatory design of agricultura decision support tools: taking account of the use situations. Agron. Sustain. Dev., 32, 899910

Chardon X., Rigolot C., Baratte C., Espagnol S., Raison C., Martin-Clouaire R., Rellier J.P., Le Gall A., Dourmad J.Y., Piquemal B., Leterme P., Paillat J.M., Delaby L., Garcia F., Peyraud J.L., Poupa J.C., Morvan T., Faverdin P., 2012. MELODIE: a whole-farm model to study the dynamics of nutrients in dairy and pig farms with crops. Animal, 6, 1711-1721.

Christensen C.M., 1997. The Innovator's Dilemma: When New Technologies Cause Great Firms to Fail. Boston: Harvard Business School Press. 225p.

COM 2003. Innovation policy: updating the Union's approach in the context of the Lisbon strategy. Brussels, 11.3.2003, 27p. [http://ec europa.eu/invest-in-research/pdf download en/innovation policy updating uni on.pdf]

Commandeur M., Le Guen R., Dourmad J.Y., Casabianca F., 2006. La diversité des styles d'élevages porcins : une approche dans les Côtes d'Armor. Journ. Rech. Porcine, 38, 247 254

Coquil X., Blouet A., Fiorelli J.L., Bazard C. Trommenschlager J.M, 2009. Conception de systèmes laitiers en agriculture biologique: une entrée agronomique. In : Numéro spécial, Elevage bio. Perez J.M. (Ed). INRA Prod. Anim., 22, 221-234.

Coulon J.B., Meynard J.M., 2011 Vers une agriculture à hautes performances environnementales : Etat des lieux des voies d'amélioration technique proposées par l'INRA. Innov. Agron., 12, 1-15.

David P.A., 1985. Clio and the economics of QWERTY. Am. Econ. Rev., 75, 332-337.

Debaeke P., Munier-Jolain N., Bertrand M., Guichard L., Nolot J.M., Faloya V., Saulas P., 2009. Iterative design and evaluation of rulebased cropping systems: methodology and case studies. Agron. Sustain. Dev., 29, 73-86.

Dedieu B., Servière G., 2011. The model of work in approaches to livestock farming systems. Options Méditerranéennes, Série A 100, 355 364

Dedieu B., Louault F., Tournadre H., Benoit M., 2008. Réponse des systèmes d'élevage innovants à la variabilité climatique : une expérimentation en production extensive ovin viande intégrant des préoccupations environnementales. In : Dedieu B., Chia E., Leclerc B., Moulin C.H., Tichit M. (Eds). L'élevage en mouvement : flexibilité et adaptation des exploitations d'herbivores. Editions Quae, Versailles, France, 161-178.

Dedieu B., Aubin J., Duteurtre G., Alexandre G., Vayssiere J., Bommel P., Faye B., 2011.
Conception et évaluation de systèmes d'élevage durables en régions chaudes. In : Numéro spécial, Elevage en régions chaudes. Coulon J.B. Lecomte P., Boval M., Perez J.M. (Eds). INRA Prod. Anim., 24, 113-129.

Dourmad J.Y., Carpentier A., Lebret B., Meunier-Salaün M.C., 2006. Le bien-être animal, un facteur d'évolution des systèmes de production porcine. Bul. Acad. Vét. de France, 159, 213-217.

Dourmad J.Y., Hassouna M., Robin P., Guingand N., Meunier-Salaün M.C., Lebret B., 2009 . Influence of pig rearing system on animal performance, manure composition and gaseous emissions. Animal, 3, 606-616.

Dumont B., Fortun-Lamothe L., Jouven M., Thomas M., Tichit M., 2013. Prospects from agroecology and industrial ecology for animal production in the $21^{\text {st }}$ century 1 . Animal, 7 , 1028-1043.

Etienne M., 2010. Companion Modelling. A participatory approach to sustainable development. Editions Quae, Versailles, France, 368p.

Etienne M., collectif ComMod., 2005. La modélisation comme outil d'accompagnement. Natures, Sciences, Sociétés, 16, 165-168.

Fagerberg J., Mowery D.C., Nelson R.R., 2005. The Oxford Handbook of Innovation. Fagerberg J., Mowery D.C., Nelson R.R., Oxford University Press., 656p.

Fares M., Magrini M.B., Triboulet P., 2012. Transition agroécologique, innovation et effets de verrouillage : le rôle de la structure organisationnelle des filières. Le cas de la filière blé dur française. Cah. Agric., 21, 34-45. [http:// dx.doi.org/10.1684/agr.2012.0539]

Farruggia A., Dumont B., Scohier A., Leroy T., Pradel P., Garel J.P., 2012. An alternative rotational stocking management designed to favour butterflies in permanent grasslands. Grass Forage Sci., 67, 136-149.

Gaigné C., Le Gallo J., Larue S., Schmitt B., 2012. Does regulation on manure land application work against agglomerations economies? Theory and evidence from the French hog sector. Am. J. Agricult. Econ., 94, 116-132.

Geels F.W., 2002. Technological transitions as evolutionary reconfiguration processes: a multilevel perspective and a case-study. Res. Pol. 31, 1257-1274. [http://dx.doi.org/10.1016/ s0048-7333(02)00062-8]

Germain K, Leterrier C., Meda B., Jurjanz S., Cabaret J., Lessire M. Jondreville C., Bonneau M., Guémené D., 2013. Elevage du poulet de chair biologique : l'utilisation du parcours influence de nombreux paramètres biotechniques $10^{\mathrm{emes}}$ Journ. Rech. Avicole et Palmipèdes à Foie Gras, 211-215.

Gouttenoire L., Cournut S., Ingrand S., 2013 Participatory modelling with farmer groups to help them redesign their livestock farming systems. Agron. Sustain. Dev., 33, 413-424.

Groot Koerkamp P.W.G., Bos A.P., 2008 Designing complex and sustainable agricultural production systems; an integrated and reflexive approach for the case of table egg production in the Netherlands. NJAS - Wageningen J. Life Sci., $55,113-138$.

Guillou M., Matheron G., 2011. Neuf milliards d'hommes à nourrir, un défi pour demain. Editions François Bourin, Paris, France, 432p. 
Hill S.B., MacRae R.J., 1995. Conceptual framework for the transition from conventional to sustainable agriculture. J. Sustain. Agric., $7,81-87$.

House of lords, 2010. Innovation in EU agriculture. European Union Committee. 19 Report, Session 2010-12. [http://www.publications.parliament.uk/pa/ld201012/ldselect/ldeucom/171/171pdf]

Hubert B., Coudel E., Coomes O., Soulard C.T., Faure G., Devautour H., 2012. En route, mais laquelle? In : Apprendre à innover dans un monde incertain ; concevoir les futurs de l'agriculture et de l'alimentation. Coudel E., Devautour H., Soulad C.T., Faure G., Hubert B. (Ed). QUAE, Paris 215-224.

Ingrand S., Devun J., Pailleux J.Y., Chauvet A., Dujour E., 2012. Les innovations en élevages bovins et ovins allaitants : analyse de résultats d'entretiens auprès d'éleveurs et de réponses d'experts à un questionnaire. Renc. Rech. Rum., 19, 393-396.

INRA-AFZ, 2004. Tables de composition et de valeur nutritive des matières premières destinées aux animaux d'élevage : porcs, volailles, bovins, ovins, caprins, lapins, chevaux, poissons. Sauvant D., Perez J.M., Tran G. (Eds), INRA, Ass. Franc. Zootech., Paris, France, 304p.

IPCC, 2006. 2006 IPCC Guidelines for national greenhouse gas inventories. Eggleston H.S., Buendia L., Miwa K., Ngara T., Tanabe K. (Eds). IGES, Japan.

Joannon A., 2004. Coordination spatiale des systèmes de culture pour la maîtrise de processus écologiques - Cas du ruissellement érosif dans les bassins versant agricoles du Pays de Caux, Haute-Normandie. Thèse de doctorat, INA P-G, Paris, 230p.

Lamine C., Bellon S., 2009. Transitions vers l'agriculture biologique. Pratiques et accompagnement vers des systèmes innovants. QuaeEducagri, Versailles-Dijon, France, 316p.

Lamine C., Meynard J.M., Bui S., Messéan A., 2010. Réductions d'intrants : des changements techniques, et après ? Effets de verrouillage et voies d'évolution à l'échelle du système agrialimentaire. Innov. Agron., 8, 121-134.

Lebret B., Prunier A., Bonhomme N., Foury A., Mormede P., Dourmad J.Y., 2011. Physiological traits and meat quality of pigs as affected by genotype and housing system. Meat Sci., 88, 14-22.

Leclerc G., Bommel P., Gibon A., Lasseur J., Morales H., 2010. Élaboration participative de modèles et de scénarios : une entrée pour analyser la coévolution des systèmes d'élevage extensif et des territoires. Cah. Agric., 19, 152-159.

Le Denmat M., Dagorn J., Dufour F. 1984. Observation sur les troupeaux de truies conduits en bandes espacées de trois semaines. Journ. Rech. Porcine, 16, 125-134.

Le Masson P., Weil B., Hatchuel A., 2006. Les processus d'innovation: Conception innovante et croissance des entreprises. Hermes Lavoisier, Paris, France, 470p.

Madelrieux S., Dedieu B., 2008. Qualification and assessment of work organisation in livestock farms. Animal, 2, 435-446.
Marousseau G., 2012. Des réponses aux crises alimentaires : étude du cas des «Poulets de Loué ». Economie et sociétés, série AG, $\mathrm{n}^{\circ} 34$.

Martel G., Dedieu B., Dourmad J.Y, 2008. Simulation of sow herd dynamics with emphasis on performance and distribution of periodic tasks events. J. Agric. Sci., 146, 365-380.

Martin G., Duru M., Magne M.A., Theau J.P., Piquet M., Felten B., Sautier M., Thenard V., 2012. Le rami fourrager: un support pour la conception de scénarios de systèmes fourragers avec des éleveurs et des conseillers. Fourrages, 210,119-128.

Meynard J.M., 2008. Produire autrement : réinventer les systèmes de cultures. In : Systèmes de culture innovants et durables. Reau R., Doré T. (Eds). Editions Educagri, 11-27.

Meynard J.M., Casabianca F., 2011. Agricultural systems and the innovation process. In: Bouche R., Derkimba A., Casabianca F. (Eds). New trends for innovation in the Mediterranean animal production. Wageningen Academic Publishers, 17-26.

Meynard J.M., Reau R., Robert D., Saulas P., 1996. Evaluation expérimentale des itinéraires techniques. In: Expérimenter sur les conduites de culture: un nouveau savoir-faire au service d'une agriculture en mutation. Paris: DERFACTA, 63-72.

Meynard J.M., Aggeri F., Coulon J.B., Habib R., Tillon J.P., 2006. Conception de systèmes agricoles innovants. Rapport à la Direction de l'INRA, p55.

Meynard J.M., Rolland B., Loyce C., Félix I., Lonnet P., 2009. Quelles combinaisons variétés / conduites pour améliorer les performances économiques et environnementales de la culture de blé tendre? Innov. Agron., 7, 29-47.

Meynard J.M., Dedieu B., Bos A.P., 2012 Re-design and co-design of farming systems. An overview of methods and practices. In: Farming Systems Research into the $21^{\text {st }}$ century: The new dynamic. Darnhofer I., Gibon D. Dedieu B. (Eds), Springer, 407-432.

Meynard J.M., Messéan A., Charlier A., Charrier F., Fares M., Le Bail M., Magrin M.B., Savini I., 2013. Freins et leviers à la diversification des cultures. Etude au niveau des exploitations agricoles et des filières. Synthèse du rapport d'étude, INRA, 52p.

Millenium Ecosystem Assessment, 2005 Ecosystems and Human Well-being: General Synthesis. Washington: Island Press [online: http://www.millenniumassessment.org/en/inde x.aspx].

Mischler P., Lheureux S., Dumoulin F., Menu P., Sene O., Hopquin J.P., Cariolle M., Reau R., Munier-Jolain N., Faloya V., Boizard H., Meynard J.M., 2009. En Picardie, 8 fermes de grande culture engagées en Production Intégrée réduisent fortement les pesticides sans baisse de marge. Le Courrier de l'Environnement., $57,73-91$

Osty P.L., 1978. L'exploitation agricole vue comme un système. Diffusion de l'innovation et contribution au développement. Bull. Tech. Inf. Min. Agric., 326, 43-49.
Perrot C., Landais E., 1993 Comment modéliser la diversité des exploitations agricoles ? Cah. Rech. Dev., 33, 24-40.

Petit M. 1975 L'adoption des innovations techniques par les agriculteurs. Plaidoyer pour un renouvellement de la théorie économique de la décision. Pour, 40, 79-91.

Reau R., Monnot L.A., Schaub A., MunierJolain N., Pambou I., Bockstaller C., Cariolle M., Chabert A., Dumans P., 2012. Les ateliers de conception de systèmes de culture pour construire, évaluer et identifier des prototypes prometteurs. Innov. Agron., 20, 5-33.

Sabatier R., Doyen L., Tichit M., 2012. Action versus result-oriented schemes in a grassland agroecocystem : a dynamic modelling approach. Plos One, 7, e33257, DOI: 10.1371.

Salembier C., Meynard J.M., 2013 Evaluation de systèmes de culture innovants conçus par des agriculteurs : un exemple dans la Pampa Argentine. Innov. Agron., 30.

Schott C., Mignolet C., Meynard J.M., 2010. Les oléoprotéagineux dans les systèmes de culture : évolution des assolements et des successions culturales depuis les années 1970 dans le bassin de la Seine. OCL - Oléagineux, Corps Gras, Lipides, 17, 276-291. [http://dx.doi.org/ $\underline{10.1684 / \mathrm{ocl} .2010 .0334]}$

Sebillotte M., 1974 Agronomie et agriculture. Essai d'analyse des tâches de l'agronome. Cahiers ORSTOM, Sr. Biol., 24, 3-25

Simon C., Etienne M., 2010. A companion modelling approach applied to forest management planning. Environ. Mod. Soft. 25, 1371 1384.

Soulard C., Thareau B., 2009. Les exploitations agricoles péri-urbaines : diversité et logiques de développement. Innov. Agron., 5, 27-40.

Steinfeld H., Gerber P., Wassenaar T., Castel V., Rosales M., de Haan C., 2006. Livestock's long shadow. Environmental issues and options. Livestock, environment and development initiative. United Nations Food Agric. Organization, Rome, Italie, 26p.

Thenail C., Joannon A., Capitaine M., Souchere V., Mignolet C., Schermann N., Di Pietro F., Pons Y., Gaucherel C., Viaud V., Baudry J., 2009. The contribution of crop-rotation organization in farms to crop-mosaic patterning at local landscape scales. Agric. Ecosys. Environ., 131, 207-219.

Thomas M., Fortun-Lamothe L., Jouven M., Tichit M., González-García E., Dourmad J.Y., Dumont B., 2014. Agro-écologie et écologie industrielle: deux alternatives complémentaires pour les systèmes d'élevage de demain. In : Numéro spécial, Quelles innovations pour quels systèmes d'élevage ? Ingrand S., Baumont R. (Eds). INRA Prod. Anim., 27, 89-100.

Vasseur C., Joannon A., Aviron S., Burel F., Meynard J.M., Baudry J., 2012. The cropping systems mosaic: How does the hidden heterogeneity of agricultural landscapes drive arthropod populations? Agric., Ecosys. Environ., 166, 3-14. 


\title{
Résumé
}

En guise d'introduction à un numéro spécial de la revue «INRA Productions Animales » sur l'innovation, cet article s'interroge sur la contribution des chercheurs au processus d'innovation. Il montre que les moteurs du changement en agriculture et en élevage appellent à un intense effort de conception innovante, et propose un panorama des démarches qui peuvent être mises en oeuvre pour ce faire : conception de novo vs pas à pas, conception assistée par modèle vs en atelier de conception, conception au niveau du système d'élevage d'un agriculteur ou au niveau du territoire. Il passe en revue les cadres d'analyse utilisés pour étudier les innovations in situ et anticiper les conditions de leur diffusion : typologies d'adoptants, verrouillage technologique, etc. Il conclut sur l'importance de revisiter la posture des chercheurs vis à vis de l'innovation.

\begin{abstract}
Innovation in animal production: renewed approaches for new issues

As an introduction to a special issue of the "Animal Production" Journal on innovation in livestock farming, this article considers the contribution of scientists in animal production, to the innovation process. It shows that the new drivers of change in agriculture and livestock farming require an intense effort towards innovative design, and it provides an overview of the approaches that can be used to do this: de novo step-by-step design, model-based design $v s$ design workshops, design at the level of the livestock system or at the landscape level. The analytical frameworks used to study in situ innovations and anticipate their dissemination are also reviewed: typologies of adopters, technological lock-in... The issue concludes on the importance of revisiting the posture of researchers towards innovation.
\end{abstract}

MEYNARD J.-M., DOURMAD J.-Y., 2014. L'innovation en élevage : de nouvelles démarches pour de nouveaux enjeux. In : Numéro spécial, Quelles innovations pour quels systèmes d'élevage ? Ingrand S., Baumont R. (Eds). INRA Prod. Anim., 27, 77.88. 